\title{
Article
}

Mycosphere

\section{A new species of Fuscoporia (Hymenochaetales, Basidiomycota) from southern China}

\section{Chen $\mathbf{Q}^{1}$ and Yuan $\mathbf{Y}$}

\author{
${ }^{1}$ Institute of Microbiology, Beijing Forestry University, Beijing 100083, China
}

Chen Q, Yuan Y 2017 - A new species of Fuscoporia (Hymenochaetales, Basidiomycota) from southern China. Mycosphere 8(6), 1238-1245, Doi 10.5943/mycosphere/8/6/9

\begin{abstract}
A new polypore species, Fuscoporia subferrea, is described from southern China based on morphological characters and phylogenetic analysis. The new species is characterized by annual, resupinate basidiocarp, hyaline, thin-walled, smooth, cylindrical basidiospores measured as 4.2-5.8 $\times 2.2-2.6 \mu \mathrm{m}$. It resembles $F$. ferrea, but differs by smaller pores $(7-10 / \mathrm{mm}$ vs. $5-7 / \mathrm{mm})$ and narrower basidiospores $(4.2-6.2 \times 2.0-2.6 \mu \mathrm{m}$ vs. $4.2-5.2 \times 2.8-3.5 \mu \mathrm{m})$. Phylogenetic analyses inferred from the ITS and nLSU sequences indicate that the new species forms a distinct lineage with strong support and is closely related to $F$. ferrea.
\end{abstract}

Key words - Hymenochaetaceae - phylogeny - taxonomy - wood-rotting fungi

\section{Introduction}

Fuscoporia Murrill was proposed by Murrill (1907) and typified by F. ferruginosa (Schrad.) Murrill. Most mycologists (Overholts 1953, Lowe 1966, Ryvarden \& Johansen 1980, Larsen \& Cobb-Poulle 1990, Ryvarden \& Gilbertson 1994) treated this genus as a synonym of Phellinus Quél. Nevertheless, Fiasson \& Niemelä (1984) recognized Fuscoporia as a monophyletic genus characterized by annual to perennial and resupinate to pileate basidiomata, a dimitic hyphal system with the encrustations on generative hyphae, the presence of hymenial setae, hyaline, thin-walled and smooth basidiospores, and the genus was confirmed by Wagner \& Fischer (2001, 2002) through their nuclear large subunit (nLSU) ribosomal RNA-based phylogeny.

Recently, some new species were reported in the genus mostly by molecular analyses (Niemelä et al. 2001, Groposo et al. 2007, Baltazar et al. 2009, Baltazar \& Gibertoni 2010, Dai 2010, Raymundo et al. 2013, Spirin et al. 2014, Pires et al. 2015).

During the survey of wood-rotting fungi in China, three unknown specimens were collected in Hainan Province, southern China, they are rather similar to Fuscoporia ferrea (Pers.) G. Cunn.but with distinct smaller pores and narrower basidiospres, and we propose a new species for these collections. To support our proposal, phylogenetic analyses on the position of the new species and related taxa were done based on internal transcribed spacer (ITS) and the large subunit nuclear ribosomal RNA gene (nLSU) sequences.

\section{Materials \& Methods}

\section{Morphological studies}


The studied specimens are deposited in the herbarium of the Institute of Microbiology, Beijing Forestry University (BJFC). Macro-morphological descriptions are based on field notes and dry herbarium specimens. Color terms follow Petersen (1996). Microscopic measurements and drawings were made from slide preparations of dried specimens stained with Cotton Blue and Melzer's reagent, by light microscopy following Dai (2010). Sections were studied at ultimate magnification $\times 1000$ using Nikon Eclipse $80 \mathrm{i}$ microscopy and phase contrast illumination. Drawings were made with the aid of drawing tube. Spores were measured in tube sections. In presenting spore size variation, $5 \%$ of measurements were excluded from each end of the range and given in parentheses. The following abbreviations were used: $\mathrm{KOH}=5 \%$ potassium hydroxide, $\mathrm{CB}$ $=$ cotton blue, $\mathrm{CB}-=$ acyanophilous, $\mathrm{IKI}=$ Melzer's reagent, IKI $-=$ neither amyloid nor dextrinoid, $\mathrm{L}=$ mean spore length (arithmetic average of all spores), $\mathrm{W}=$ mean spore width (arithmetic average of all spores), $\mathrm{Q}=$ variation in the $\mathrm{L} / \mathrm{W}$ ratios between specimens studied, $\mathrm{n}$ $(\mathrm{a} / \mathrm{b})=$ number of spores (a) measured from given number of specimens (b).

\section{DNA extraction and sequencing}

A CTAB rapid plant genome extraction kit (Aidlab Biotechnologies Co., Ltd., Beijing, China) was used to extract total genomic DNA from dried specimens following the manufacturer's instructions with some modifications (Chen et al. 2015, 2016). ITS regions were amplified with primers ITS4 and ITS5 (White et al. 1990), and the nLSU with primers LR0R and LR7. The PCR procedure for ITS was as follows: $6 \mathrm{v}$ initial denaturation at $95^{\circ} \mathrm{C}$ for $3 \mathrm{~min}$, followed by 35 cycles at $94{ }^{\circ} \mathrm{C}$ for $40 \mathrm{~s}, 54{ }^{\circ} \mathrm{C}$ for $45 \mathrm{~s}$ and $72{ }^{\circ} \mathrm{C}$ for $1 \mathrm{~min}$, and a final extension of $72{ }^{\circ} \mathrm{C}$ for $10 \mathrm{~min}$. The PCR procedure for nLSU was as follows: initial denaturation at $94{ }^{\circ} \mathrm{C}$ for $1 \mathrm{~min}$, followed by 35 cycles at $94{ }^{\circ} \mathrm{C}$ for $1 \mathrm{~min}, 50{ }^{\circ} \mathrm{C}$ for $1 \mathrm{~min}$ and $72{ }^{\circ} \mathrm{C}$ for $1.5 \mathrm{~min}$, and a final extension of $72{ }^{\circ} \mathrm{C}$ for $10 \mathrm{~min}$. The PCR products were purified and sequenced in Beijing Genomics Institute, China, with the same primers. All newly generated sequences were deposited at GenBank and listed in Table 1.

Besides the sequences generated from this study, other reference taxa for our phylogenetic analysis were selected from GenBank, and the original publications of the phylogenetic analyses contributing the sequences were referenced in Table 1. Sequences were aligned in MAFFT 6 (Katoh \& Toh 2008) using the "G-INS-I" strategy and manually adjusted in BioEdit (Hall 1999).

\section{Phylogenetic analyses}

Maximum Likelihood (ML) analysis was applied to the combined dataset. It was conducted with RAxML-HPC2 on Abe through the Cipres Science Gateway involved 1000 replicates under the GTRGAMMA model, with all model parameters estimated by the program. In addition 1000 rapid bootstrap replicates were run with the GTRCAT model.

Maximum parsimony (MP) analysis was also applied to the combined dataset as in Zhou et al. (2016b). Tree construction was performed in PAUP* version 4.0b10 (Swofford 2002). All characters were equally weighted and gaps were treated as missing data. Trees were inferred using the heuristic search option with TBR branch swapping and 1000 random sequence additions. Maxtrees were set to 5000, branches of zero length were collapsed and all parsimonious trees were saved. Clade robustness was assessed using a bootstrap (BT) analysis with 1000 replicates (Felsenstein 1985). Descriptive tree statistics tree length (TL), consistency index (CI), retention index (RI), rescaled consistency index (RC), and homoplasy index (HI) were calculated for each Maximum Parsimonious Tree (MPT) generated.

Bayesian inference (BI) was applied to the combined dataset too. Substitution models suitable for each partition in the dataset were determined using Akaike Information Criterion (AIC) implemented in MrModeltest2.3 (Posada \& Crandall 1998, Nylander 2004). The GTR+I+G model was estimated as the best-fit evolution models for all partition in the combined dataset. BI was calculated with MrBayes3.1.2 (Ronquist \& Huelsenbeck 2003) with GTR+I+G model of DNA substitution and an invgamma distribution rate variation across sites. Four Markov chains were run for 2 runs from random starting trees for 2 million generations of the combined ITS and nLSU dataset and sampled every 100 generations. The burn-in was set to discard the first $25 \%$ of the 
trees. A majority rule consensus tree of all remaining trees was calculated. Branches that received bootstrap support for maximum parsimony (MP), maximum likelihood (BS) and Bayesian posterior probabilities (BPP) greater than or equal to $75 \%$ (MP/BS) and 0.95 (BPP) were considered as significantly supported, respectively.

\section{Results}

\section{Phylogenetic analyses}

The combined ITS+nLSU dataset included sequences from 39 fungal specimens representing 15 species. The dataset had an aligned length of 2253 characters, of which 1722 are constant, 40 are variable but parsimony-uninformative, and 491 are parsimony-informative. Maximum Likelihood analysis resulted in a best tree, which is shown in Fig 1. Maximum parsimony analysis yielded 4 similar topologies $(\mathrm{TL}=1177, \mathrm{CI}=0.650, \mathrm{RI}=0.873, \mathrm{RC}=0.568$, $\mathrm{HI}=0.350$ ). Bayesian also resulted in a similar consensus tree with 1 million generation and an average standard deviation of split frequencies $=0.007745$. The outgroup selected for ITS $+n L S U$ analysis was Coniferiporia weirii (Murrill) L.W. Zhou \& Y.C. Dai, and Coniferiporia sulphurascens (Pilát) L.W. Zhou \& Y.C. Dai because Coniferiporia was a sister group of Fuscoporia (Zhou et al. 2016a).

In the phylogeny (Fig. 1) inferred from the combined ITS+nLSU sequences shows that the three newly sequenced specimens are clustered into a lineage with high support (100/100/1.00), which is closely related to Fuscoporia ferrea.

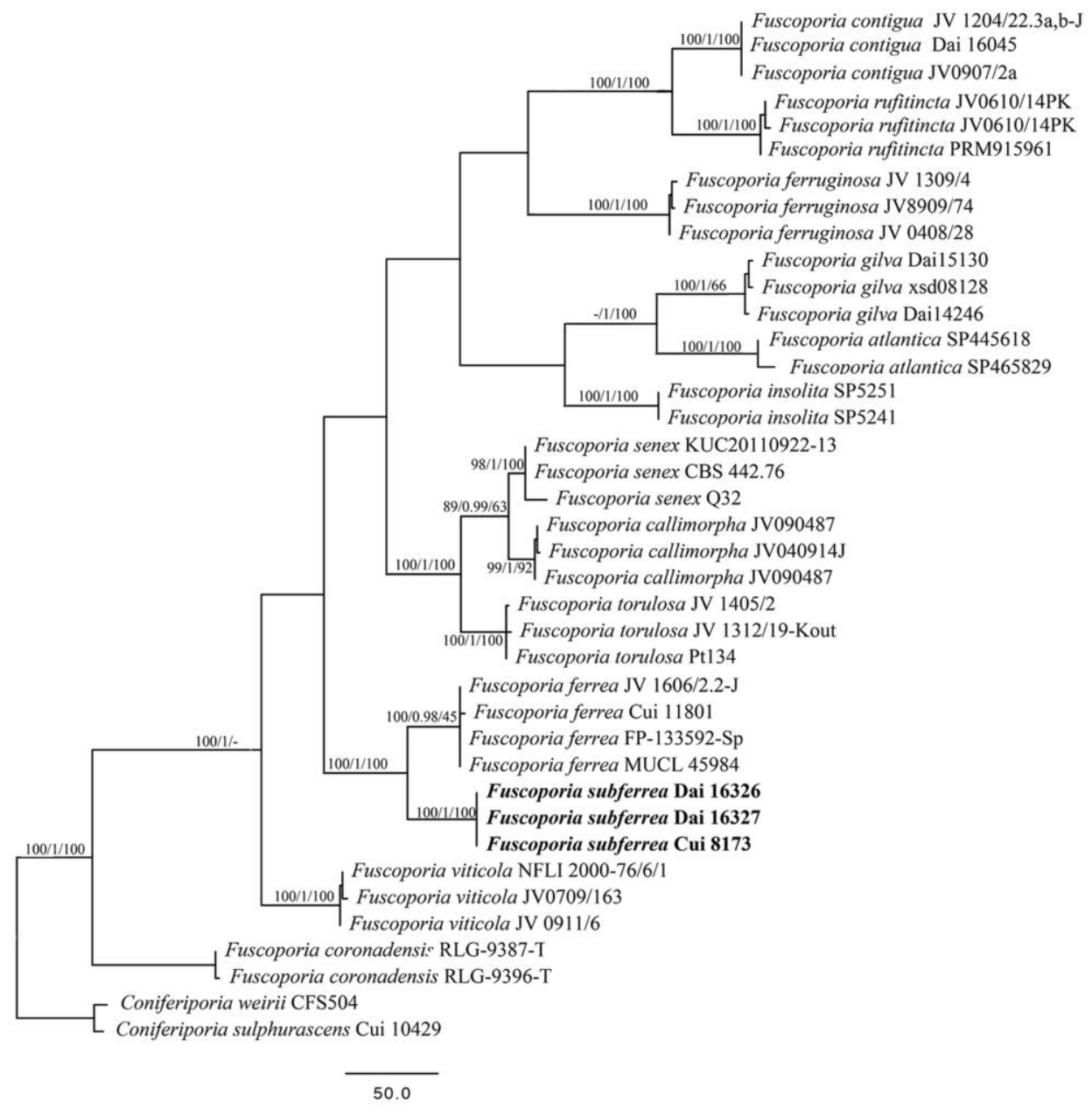

Figure 1 - Phylogeny of Fuscoporia subferrea and related species generated by maximum likelihood analysis based on combined ITS and nLSU sequences. Branches are labeled with maximum likelihood bootstrap value higher than 50\%, parsimony bootstrap value higher than 50\%, and Bayesian posterior probabilities more than 0.95 . 


\section{Taxonomy}

Fuscoporia subferrea Q. Chen \& Yuan Yuan, sp. nov.

Figs $2-3$

MycoBank - MB 819479

Holotype - China, Hainan Province, Baisha County, Yinggeling Nature Reserve, on fallen angiosperm branch, 17 November 2015, Dai 16327 (BJFC020414, holotype).

Etymology - Subferrea (Lat.) - referring to the morphological resemblance to Fuscoporia ferrea.

Fruiting body - Basidiocarps annual, resupinate, inseparable, without odour or taste and corky when fresh, light-weight and hard corky when dry, up to $26 \mathrm{~cm}$ long, $3 \mathrm{~cm}$ wide and $2 \mathrm{~mm}$ thick at centre. Pore surface mouse-grey to ash-grey when fresh, more or less fawn, cracked with age; sterile margin distinct, dark reddish brown when dry, up to $1.5 \mathrm{~mm}$ wide; pores circular, 7-10 per mm; dissepiments thin, entire, abundant setae seen in tube cavities (under lens). Subiculum dull brown, corky, about $0.4 \mathrm{~mm}$ thick. Tubes yellowish brown, paler contrasting with pores and subiculum, hard corky, up to $1.6 \mathrm{~mm}$ long.

Hyphal structure - Hyphal system dimitic; generative hyphae simple septate; tissue darkening but otherwise unchanged in $\mathrm{KOH}$.

Subiculum - Generative hyphae infrequent, hyaline, thin- to slightly thick-walled, occasionally branched, frequently simple septate, 2.0-2.6 $\mu \mathrm{m}$ in diam; skeletal hyphae dominant, rust-brown, thick-walled with a narrow to medium lumen, unbranched, flexuous, interwoven, 2.4$3.2 \mu \mathrm{m}$ in diam.

Tubes - Generative hyphae infrequent, mostly present at subhymenium, hyaline, thin-walled, frequently branched and simple septate, $1.8-2.4 \mu \mathrm{m}$ in diam, some of them at dissepiment edges and in hymenium encrusted; skeletal hyphae dominant, yellowish brown, thick-walled with a narrow to medium lumen, unbranched, more or less straight, interwoven, 2.2-3.0 $\mu \mathrm{m}$ in diam. Setae frequent, mostly originating from hymenium, subulate, dark brown, thick-walled, 18-34 $\times 4-7 \mu \mathrm{m}$; fusoid cystidioles frequent, hyaline and thin-walled, sometimes covered with crystals; basidia barrel-shaped, with four sterigmata and a simple septum at the base, 9.5-11 $\times 4.8-6.2 \mu \mathrm{m}$; basidioles dominating in hymenium, in shape similar to basidia, but slightly smaller.

Spores - Basidiospores cylindric, hyaline, thin-walled, smooth, usually glued in tetrads, IKI-, $\mathrm{CB}-$, sometimes with one or two guttules, (4.0-)4.2-6.2(-6.4) $\times(1.8-) 2.0-2.6(-2.8) \mu \mathrm{m}, \mathrm{L}=5.11$ $\mu \mathrm{m}, \mathrm{W}=2.28 \mu \mathrm{m}, \mathrm{Q}=2.15-2.27(\mathrm{n}=60 / 2)$.

Additional specimens examined (paratypes) - China, Hainan Province, Baisha County, Yinggeling Nature Reserve, on fallen angiosperm branch, 17 November 2015, Dai 16326 (BJFC020413); Yunnan Province, Baoshan County, Gaoligong Mountain Nature Reserve, 25 October 2009, Cui 8173 (BJFC006662).

\section{Discussion}

According to the phylogenetic analysis (Fig. 1), four specimens of $F$. ferrea from USA (JV 1606/2.2-J, FP-133592-Sp), France (MUCL 45984) and northern China (Cui 11801) are closely related to the $F$. subferra, but the two species are in two distinct lineages in our phylogeny.

Fuscoporia subferrea is morphologically similar to $F$. ferrea in sharing annual, resupinate basidiocarps and cylindric basidiospores, but the latter has distinctly wider basidiospores (4.2-5.2 $\times$ 2.8-3.5 $\mu \mathrm{m}$ ) and larger pores (5-7 per $\mathrm{mm}$ ). In addition, these two species have different distributions in China: F. subferrea mainly distributes in southern China, while $F$. ferrea has a distribution in northern China. $F$. suferrea sometimes is confused with $F$. ferruginosa, but the latter has ellipsoid basidiospores and abundant mycelial setae at the sterile margin. F. chrysea (Lév.) Baltazar \& Gibertoni is similar to $F$. subferrea by resupinate fruiting bodies and tiny pores (9-10 per $\mathrm{mm}$ ), but it has shorter basidiospores (3.5-4 × 2.5-3 $\mu \mathrm{m}$, Baltazar \& Gibertoni 2010). 
Table 1 Taxa and GenBank accession numbers for ITS and nLSU sequences used in the phylogenetic analyses.

\begin{tabular}{|c|c|c|c|c|}
\hline \multirow{2}{*}{ Species } & \multirow{2}{*}{ Sample no. } & \multirow{2}{*}{ Locality } & \multicolumn{2}{|c|}{ GenBank accession no. } \\
\hline & & & ITS & nLSU \\
\hline Fuscoporia atlantica & SP445618 & Brazil & KP058515 & KP058517 \\
\hline Fuscoporia atlantica & SP465829 & Brazil & KP058514 & KP058516 \\
\hline Fuscoporia callimorpha & JV090487 & USA & JF692190 & - \\
\hline Fuscoporia callimorpha & JV090487 & USA & JF692191 & - \\
\hline Fuscoporia callimorpha & JV040914J & USA & JF692193 & - \\
\hline Fuscoporia contigua & JV0907/2a & USA & JQ794547 & - \\
\hline Fuscoporia contigua & Dai 16045 & USA & KX961105 a & KY189105 a \\
\hline Fuscoporia contigua & JV1204/22.3a,b-J & USA & KX961104 ${ }^{\mathrm{a}}$ & KY189104 ${ }^{\mathrm{a}}$ \\
\hline Fuscoporia coronadensis & RLG-9387-T & USA & JX110073 & JX110117 \\
\hline Fuscoporia coronadensis & RLG-9396-T & USA & JX110074 & JX110118 \\
\hline Fuscoporia ferrea & JV 1606/2.2-J & USA & KX961100 a & KY $189100^{\mathrm{a}}$ \\
\hline Fuscoporia ferrea & Cui 11801 & China & KX961101 ${ }^{\text {a }}$ & KY189101 a \\
\hline Fuscoporia ferrea & MUCL 45984 & France & KX961112 a & KY189112 \\
\hline Fuscoporia ferrea & FP-133592-Sp & USA & KU139189 & KU139259 \\
\hline Fuscoporia ferruginosa & JV1309/4 & Slovakia & KX961102 & KY189102 a \\
\hline Fuscoporia ferruginosa & JV0408/28 & Czech & KX961103 a & KY189103 a \\
\hline Fuscoporia ferruginosa & JV8909/74 & Germany & JQ794573 & - \\
\hline Fuscoporia gilva & Dai14246 & China & KX961108 a & KY189109 a \\
\hline Fuscoporia gilva & Dai 15130 & China & KX961109 a & KY189108 a \\
\hline Fuscoporia gilva & xsd08128 & China & FJ481039 & - \\
\hline Fuscoporia insolita & SP5251 & Russia & KJ677113 & - \\
\hline Fuscoporia insolita & SP5241 & Russia & KJ677114 & - \\
\hline Fuscoporia rutincta & PRM915961 & USA & GU594160 & - \\
\hline Fuscoporia rutincta & JV0610/14PK & Belize & JQ794579 & - \\
\hline Fuscoporia rutincta & JV0610/14PK & Belize & JQ794580 & - \\
\hline Fuscoporia senex & CBS 442.76 & Korea & AY558647 & - \\
\hline Fuscoporia senex & KUC20110922-13 & Korea & JX463658 & $\mathrm{JX} 463652$ \\
\hline Fuscoporia senex & Q32 & China & KC414230 & - \\
\hline Fuscoporia subferrea & Dai 16326 & China & KX961097 a & KY053472a \\
\hline Fuscoporia subferrea & Dai 16327 & China & KX961098 a & KY053473 ${ }^{\mathrm{a}}$ \\
\hline Fuscoporia subferrea & Cui 8173 & China & KX961099 a & KY189111 a \\
\hline Fuscoporia torulosa & JV $1405 / 2$ & Czech & KX961106 ${ }^{a}$ & KY189106 ${ }^{\mathrm{a}}$ \\
\hline Fuscoporia torulosa & JV 1312/19-Kout & Spain & KX961107 a & KY189107 a \\
\hline Fuscoporia torulosa & Pt134 & Italy & EF068139 & - \\
\hline Fuscoporia viticola & JV0911/6 & Czech & KX961110 a & KY189110 a \\
\hline Fuscoporia viticola & NFLI $2000-76 / 6 / 1$ & - & JQ358814 & - \\
\hline Fuscoporia viticola & JV0709/163 & USA & JQ794583 & - \\
\hline $\begin{array}{l}\text { Coniferiporia } \\
\text { sulphurascens }\end{array}$ & Cui 10429 & China & KR350565 & KR350555 \\
\hline Coniferiporia weirii & CFS504 & Canada & AY829341 & AY829345 \\
\hline
\end{tabular}

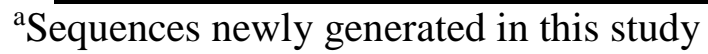

\section{Acknowledgements}

We express our gratitude to Profs. Yu-Cheng Dai and Bao-Kai Cui (BJFC, China) for allowing us to study their specimens. The research is supported by the National Natural Science Foundation of China (project No. 31530002). 

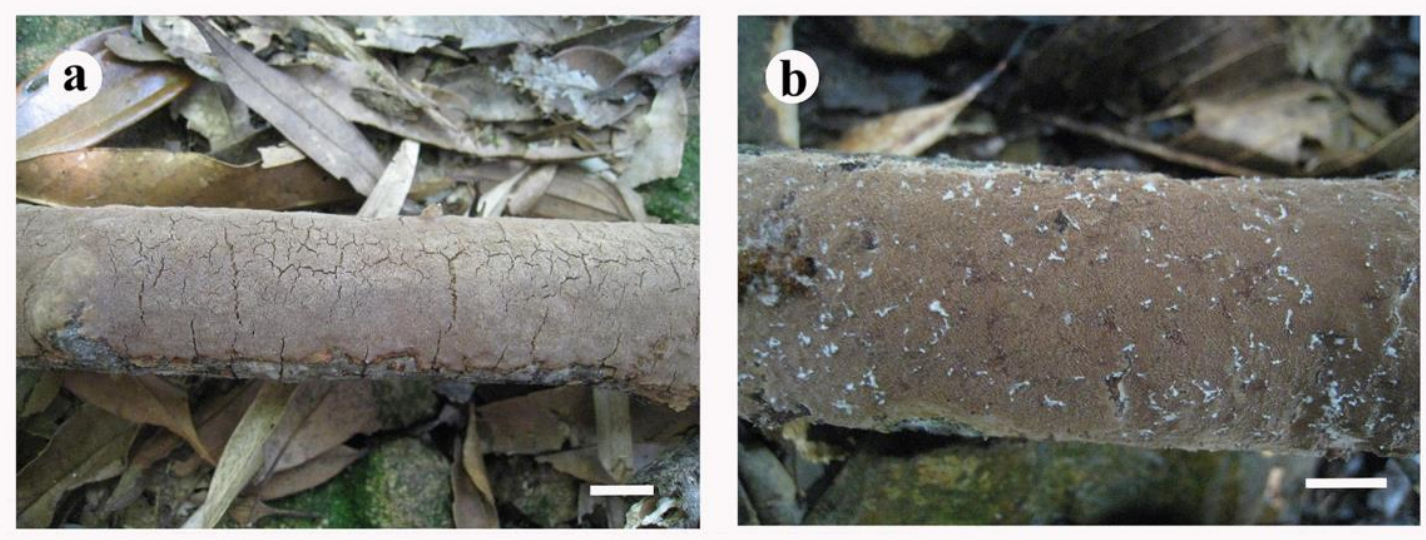

Figure 2 - Basidiocarps of Fuscoporia subferrea. a: holotype, Dai 16327; b: Dai 17159. Scale bars $=10 \mathrm{~mm}$.

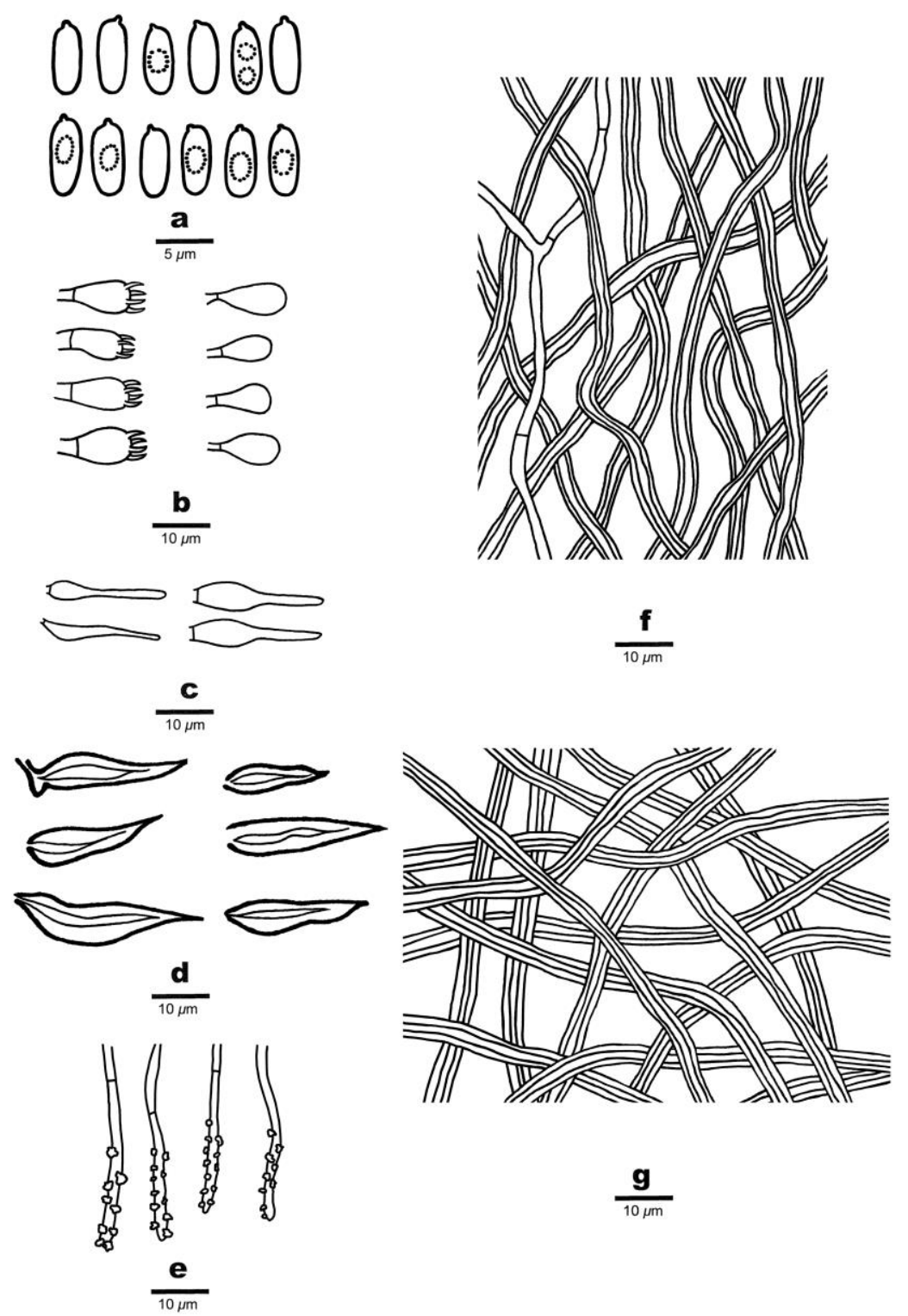

Figure 3 - Microscopic structures of Fuscoporia subferrea. (the holotype, Dai 16327). a: basidiospores; b: basidia and basidioles; c: cystidioles; d: setae; e: generative hyphae at dissepiment edge; f: hyphae from trama; g: hyphae from subiculum. 


\section{References}

Baltazar JM, Trierveiler-Pereira L, Loguercio-Leite C, Ryvarden L. 2009 - Santa Catarina Island mangroves 3: a new species of Fuscoporia. Mycologia 100, 859-863.

Baltazar JM, Gibertoni TB. 2010 - New combinations in Phellinus s.l. and Inonotus s.l. Mycotaxon 111, 205-208.

Chen JJ, Cui BK, Zhou LW, Korhonen K, Dai, YC. 2015 - Phylogeny, divergence time estimation, and biogeography of the genus Heterobasidion (Basidiomycota, Russulales). Fungal Diversity 71, 185-200.

Chen JJ, Cui BK, Dai YC. 2016 - Global diversity and molecular systematics of Wrightoporia s. 1. (Russulales, Basidiomycota). Persoonia 37, 21-36.

Dai YC. 2010 - Hymenochaetaceae (Basidiomycota) in China. Fungal Diversity 45, 131-343.

Felsenstein J. 1985 - Confidence intervals on phylogenetics: an approach using bootstrap. Evolution 39, 783-791.

Fiasson JL, Niemalä T. 1984 - The Hymenochaetales: a revision of the European poroid taxa. Karstenia 24, 14-28.

Groposo C, Loguercio-Leite C, Góes-Neto. 2007 - Fuscoporia (Basidiomycota, Hymenochaetales) in southern Brazil. Mycotaxon 101, 55-63.

Hall TA. 1999 - Bioedit: a user-friendly biological sequence alignment editor and analysis program for Windows 95/98/NT. Nucleic Acids Symposium Series 41, 95-98.

Katoh K, Toh H. 2008 - Recent developments in the MAFFT multiple sequence alignment program. Briefings in Bioinformatics 9, 286-298.

Larsen MJ, Cobb-Poulle LA. 1990 - Phellinus (Hymenochaetaceae): A survey of the world taxa. Synopsis Fungorum 3, 1-206.

Lowe JL. 1966 - Polyporaceae of North America The genus Poria. State University College of Forestry at Syracuse University, Syracuse, New York, 183 pp.

Murrill WA. 1907 - (Agaricales) Polyporaceae (pars). North American Flora 9, 1-131.

Niemelä T, Wagner T, Fischer M, Dai YC. 2001 - Phellopilus gen. nov. and its affinities within Phellinus s. lato and Inonotus s. lato (Basidiomycetes). Annales Botanici Fennici 38, 51-62.

Nylander J. 2004 - MrModeltest v2. Program distributed by the author. Evolutionary Biology Centre, Uppsala University.

Overholts LD. 1953 - The Polyporaceae of the United States, Alaska and Canada. Ann Arbor: University of Michigan Press.

Petersen JH. 1996 - The Danish Mycological Society's colour-chart. Foreningen til Svampekundskabens Fremme, Greve, 6 pp.

Pires RM, Motatovásquez V, De Gugliotta AM. 2015 - Fuscoporia atlantica sp. nov., a new polypore from the Brazilian Atlantic Rainforest. Mycotaxon 130, 843-855.

Posada D, Crandall KA. 1998 - Modeltest: testing the model of DNA substitution. Bioinformatics $14,817-818$.

Raymundo T, Valenzuela R, Bautista-Hernández S, Esqueda M et al. 2013 - Hymenochaetaceae from Mexico 6. A new Fuscoporia species from the Sonoran desert. Mycotaxon 125, 37-43.

Ronquist F, Huelsenbeck JP. 2003 - MrBayes 3: bayesian phylogenetic inference under mixed models. Bioinformatics 19, 1572-1574.

Ryvarden L, Johansen I. 1980 - A preliminary polypore flora of East Africa. Fungiflora, Oslo, 336 pp.

Ryvarden L, Gilbertson RL. 1994 - European polypores 2. Synopsis Fungorum 7, 394-743.

Spirin V, Vlasák J, Niemelä T. 2014 - Fuscoporia insolita (Hymenochaetales, Basidiomycota), a new species from Russian Far East. Annales Botanici Fennici 51, 403-406.

Swofford DL. 2002 - PAUP*: Phylogenetic analysis using parsimony (*and other methods). Version 4.0b10. Sinauer Associates, Massachusetts, 142 pp.

White TJ, Bruns T, Lee S, Taylor J. 1990 - Amplification and direct sequencing of fungal ribosomal RNA genes for phylogenetics. In: Innis, M.A., Gefand, D.H., Sninsky, J.J. \& 
White, J.T. (Eds.) PCR Protocols: a guide to methods and applications (eds.). Academic Press, San Diego, pp. 315-322.

Wagner T, Fischer M. 2001 - Natural groups and a revised system for the European poroid Hymenochaetales (Basidiomycota) supported by nLSU rDNA sequence data. Mycological Research 105, 773-782.

Wagner T, Fischer M. 2002 - Proceedings towards a natural classification of the worldwide taxa Phellinus s.l. and Inonotus s.l., and phylogenetic relationships of allied genera. Mycologia 94, 998-1016.

Zhou LW, Vlasák J, Dai YC. 2016a - Taxonomy and phylogeny of Phellinidium (Hymenochaetales, Basidiomycota): a redefinition and the segregation of Coniferiporia gen. nov. for forest pathogens. Fungal Biology 120, 988-1001.

Zhou LW, Vlasák J, Decock C, Assefa A et al. 2016b - Global diversity and taxonomy of the Inonotus linteus complex (Hymenochaetales, Basidiomycota): Sanghuangporus gen. nov., Tropicoporus excentrodendri and T. guanacastensis gen. et spp. nov., and 17 new combinations. Fungal Diversity 77, 335-347. 